Check for updates

Cite this: RSC Adv., 2021, 11, 22565

\title{
Structure, phase transition and properties of the one-dimensional antiferromagnet $\mathrm{Cu}(2,6-$ dimethylpyrazine) $\mathrm{Br}_{2} \uparrow$
}

\author{
Fei Ding, ${ }^{a}$ Chuanlu Yang, ${ }^{a}$ Xiangnan Gong, ${ }^{b}$ Hui Zheng, ${ }^{c}$ Xiaoyu Zhou, ${ }^{a}$ Lingli Li, ${ }^{a}$ \\ Lichun Zhang, ${ }^{a}$ Dehua Wang ${ }^{a}$ and Bingying Pan (D) *a
}

\begin{abstract}
We report the crystal structure and properties of the one-dimensional $S=1 / 2$ antiferromagnet $\mathrm{Cu}(2,6$ dimethylpyrazine) $\mathrm{Br}_{2}$ with strong intra-chain exchange. At room temperature, its linear spin chains are formed by $\mathrm{Cu}^{2+}$ ions via the non-bonding $\mathrm{Br} \cdots \mathrm{Br}$ contacts. Interestingly, a phase transition from Pmmn to $P 21 / n$ structure occurs at $T_{\mathrm{S}} \approx 248 \mathrm{~K}$ below which the $\left[\mathrm{CuBr}_{2}\right]_{n}$ spin chains become non-linear and the magnetic susceptibility abruptly increases, reflecting the weakening of antiferromagnetic exchange strength. This result evidences the Goodenough-Kanamori rules which claim that a linear superexchange pathway produces stronger antiferromagnetic coupling. From magnetic susceptibility measurements we find the average intra-chain exchange strength is $J / k_{B} \approx-88.18 \mathrm{~K}$ in the low temperature phase. Both magnetic and specific measurements show the absence of magnetic ordering down to $2 \mathrm{~K}$, implying the excellent magnetic one-dimensionality of $\mathrm{Cu}\left(2,6\right.$-dimethylpyrazine) $\mathrm{Br}_{2}$. We also performed ultra-violet (UV) absorption and photoluminescence measurements which give a semiconducting band gap $\Delta \approx 2.79 \mathrm{eV}$ which is consistent with theoretical calculations.
\end{abstract}

Received 24th March 2021

Accepted 21st June 2021

DOI: $10.1039 / \mathrm{d} 1 \mathrm{ra02318g}$

rsc.li/rsc-advances past decades, various copper(II) coordination complexes have been reported as material realizations of $1 \mathrm{D}$ quantum spin models. ${ }^{1-3}$ The properties of $1 \mathrm{D}$ magnets are directly affected by the superexchange pathways which are dependent on the concrete crystalline structure. Especially, the non-bonding contacts between halide ions $(\mathrm{Cu}-\mathrm{X} \cdots \mathrm{X}-\mathrm{Cu}, \mathrm{X}=\mathrm{Br}, \mathrm{Cl})$ represents an important type of superexchange pathways and have been widely studied. ${ }^{1,3}$ The sign and strength of the magnetic interaction via such a two-halide bridge depend on various parameters such as the geometry of the chains and the distance of the bridging halide ions. ${ }^{1,5}$ In fact, all related variables in the coordination environment have potential influence on the properties of $1 \mathrm{D}$ magnetism. ${ }^{1}$ For example, studies show that the $\mathrm{Br} \cdots \mathrm{Br}$ contacts propagate stronger exchanges than their $\mathrm{Cl} \cdots \mathrm{Cl}$ counterparts in all cases. ${ }^{6-8}$ Upon to date, a full description on the magnetic interaction via the two-halide bridge is still lacking.

The pyrazine-based compound $\mathrm{Cu}$ (pyrazine) $\left(\mathrm{NO}_{3}\right)_{2}$ is a model 1D Heisenberg antiferromagnet and has been extensively studied by neutron scattering, ${ }^{9}$ muon-spin relaxation $(\mu \mathrm{SR}),{ }^{10}$ nuclear magnetic resonance (NMR) spectroscopy, ${ }^{11,12}$ and thermal transport. ${ }^{13}$ Substituting pyrazine with related ligands such as phenazine, quinoxaline, methylpyrazine, and chloropyrazine also result in a variety of linear spin chains, but the magnetic super-exchange through pyrazine ring is weak with $J / k_{\mathrm{B}}$ smaller than $-10.3 \mathrm{~K} \cdot{ }^{14}$ By using $\mathrm{Br}$ as anions, a derivative $1 \mathrm{D}$ antiferromagnet $\mathrm{Cu}$ (2,5-dimethylpyrazine) $\mathrm{Br}_{2}$ exhibit a exceptionally strong exchange with $J / k_{\mathrm{B}}=-234 \mathrm{~K}$ and 
the authors demonstrate the dominate antiferromagnetic exchange propagate through the non-bonding $\mathrm{Cu}-\mathrm{Br} \cdots \mathrm{Br}-\mathrm{Cu}$ pathway. ${ }^{15}$ Such strong exchange is also very unusual among the 1D magnets mediated by the two bromide contacts. ${ }^{1}$ It is of great interest to investigate related compounds in order to explore the mechanism of enhanced superexchange in this twohalide bridged spin chain system.

$\mathrm{Cu}$ (2,6-dimethylpyrazine) $\mathrm{Br}_{2}$ (1) is an analog compound of $\mathrm{Cu}(2,5$-dimethylpyrazine $) \mathrm{Br}_{2}$ but its structure has not been reported in literature. The preliminary magnetic susceptibility of 1 was reported by Inman et al. in 1972 on powder sample and the data was explained by an antiferromagnetic spin chain model with $J=-47.5 \mathrm{~K} .^{2}$ However, no magnetic susceptibility data is available in a wide temperature range above $100 \mathrm{~K}$, leaving large uncertainty on the data fitting result. ${ }^{2}$

In this work, we report the structure, phase transition, magnetism, specific heat and optical properties of $\mathbf{1}$. At room temperature, $\mathbf{1}$ is featured by $\left[\mathrm{CuBr}_{2}\right]_{n}$ spin chains, similar to $\mathrm{Cu}(2,5$-dimethylpyrazine $) \mathrm{Br}_{2}$. Surprisingly, an unique phase transition from Pmmn to $P 21 / n$ structure occurs at $T_{\mathrm{S}}=248 \mathrm{~K}$, resulting in significant deformation of the spin chains at the low temperature (LT) phase. This structural transition is accompanied by a sharp jump in magnetic susceptibility across $T_{\mathrm{S}}$, indicative of the weakening of the intra-chain exchange strength once entering the LT phase. By fitting the LT susceptibility data by a uniform spin chain model we find the average exchange strength is $-88.18 \mathrm{~K}$, this strength is comparable to that of $\mathrm{Cu}(2,5$-dimethylpyrazine $) \mathrm{Br}_{2}$. The specific heat data do not show any feature of magnetic ordering down to $2 \mathrm{~K}$, indicating excellent $1 \mathrm{D}$ magnetism of $\mathbf{1}$. Our work reveals $\mathbf{1}$ is an unique 1D system that shows linear to non-linear transition in spin chains which has not been found in other copper(II) coordination complexes at ambient pressure. This system thus provides a novel platform to study the mechanism of spin chain geometry on the two-halide bridge mediated magnetic superexchange.

\section{Experimental}

\section{Synthesis}

Single crystals of $\mathrm{Cu}(2,6$-dimethylpyrazine $) \mathrm{Br}_{2}$ were synthesized by conventional diffusion method. $4 \mathrm{mmol}$ 2,6-methylpyrazine (>99\%, Adamas-beta) and $4 \mathrm{mmol}$ copper bromide (>99\%, Adamas-beta) were loaded into the $250 \mathrm{~mL}$ and $50 \mathrm{~mL}$ beakers, respectively. The $50 \mathrm{~mL}$ beaker was put inside the larger one and alcohol was slowly introduced till the alcohol level is far up beyond the edge of the $50 \mathrm{~mL}$ beaker. After about one week, black single crystals were formed. The typical single crystal size is $3 \mathrm{~mm} \times 0.25 \mathrm{~mm} \times 0.1 \mathrm{~mm}$. Anal. calc.: C, $21.72 \% ; \mathrm{H}$, $2.41 \%$; N, 8.45\%; found: C, $21.76 \%$;, $2.62 \%$;, $8.44 \%$.

\section{X-ray diffraction}

We used the three-circle diffractometer (Mo $\mathrm{K}_{\alpha}$ radiation, $\lambda=$ $0.71073 \AA$ ) for the X-ray diffraction of $\mathrm{Cu}(2,6$-dimethylpyrazine) $\mathrm{Br}_{2}$ single crystal. High quality diffraction data were collected at 300 and $240 \mathrm{~K}$ and the crystal structures were solved by the direct method and refined via full-matrix least-square techniques using the Olex2 and SHELXL program package. ${ }^{16,17}$ The resulted cell parameters, selective atomic coordinates, bond lengths and bond angles are listed in Tables 1 and 2. Crystallographic data of $\mathrm{Cu}(2,6$-dimethylpyrazine $) \mathrm{Br}_{2}$ have been deposited at the Cambridge Crystallographic Data Center (CCDC No. $2070208(T=300 \mathrm{~K})$ and $2070209(T=240 \mathrm{~K})$ ).

\section{Elemental analysis, photoluminescence, and UV absorption measurements}

Elemental analysis were determined on an Vario EL cube analyzer. Photoluminescence (PL) measurements were conducted with a $325 \mathrm{~nm}$ He-Cd laser as the pump source (Kimmon Electric, Ltd., Saitama, Japan) and a monochromator (SR-500i-A, Andor Technology, Belfast, UK) working at room temperature. The characteristic absorption peak was measured by a Shimadzu UV-2550 spectrophotometer.

\section{Magnetic susceptibility and specific heat measurements}

The magnetic susceptibility properties were measured by a MPMS SQUID magnetometer (Quantum Design) with magnetic field up to $7 \mathrm{~T}$. The measured temperature range is from 300 to $2 \mathrm{~K}$. Specific heat was measured by a Physical Property Measurement System (PPMS, Quantum Design) down to $2 \mathrm{~K}$.

\section{Results and discussion}

Magnetic susceptibility of $\mathbf{1}$ on powder sample was measured under 1000 Oe from 300 to $2 \mathrm{~K}$ (Fig. 1(a)). A abrupt jump in $\chi$ appears around $T_{\mathrm{S}}=248 \mathrm{~K}$ which corresponds to a phase transition from high temperature (HT) phase to a low temperature (LT) phase. Fig. 1(b) is a detailed measurement around $T_{\mathrm{S}}$.

Table 1 Crystal data and structure refinement of $\mathrm{Cu}(2,6$-dimethylpyrazine) $\mathrm{Br}_{2}$ at 300 and $240 \mathrm{~K}$

\begin{tabular}{|c|c|c|}
\hline Temperature (K) & $300(2)$ & $240(2)$ \\
\hline Empirical formula & $\left(\mathrm{C}_{6} \mathrm{H}_{8} \mathrm{~N}_{2}\right) \mathrm{CuBr}_{2}$ & $\left(\mathrm{C}_{6} \mathrm{H}_{8} \mathrm{~N}_{2}\right) \mathrm{CuBr}_{2}$ \\
\hline Formula weight & 331.50 & 331.50 \\
\hline Space group & Pmmn & $P 21 / n$ \\
\hline Crystal system & Orthorhombic & Monoclinic \\
\hline Wavelength & $0.71073 \AA$ & $0.71073 \AA$ \\
\hline \multirow[t]{5}{*}{ Lattice parameters } & $a=8.3552(9) \AA$ & $a^{*}=10.2576(5) \AA$ \\
\hline & $b=8.5782(11) \AA$ & $b^{*}=8.3613(5) \AA$ \\
\hline & $c=6.6959(7) \AA$ & $c^{*}=11.2178(5) \AA$ \\
\hline & $\alpha=\beta=\gamma=90^{\circ}$ & $\alpha^{*}=\gamma^{*}=90^{\circ}$ \\
\hline & & $\beta^{*}=102.982(2)^{\circ}$ \\
\hline$Z$ & 2 & 4 \\
\hline$\mu\left(\mathrm{mm}^{-1}\right)$ & 10.541 & 10.791 \\
\hline$\theta_{\min } / \theta_{\max }\left(^{\circ}\right)$ & $6.808 / 77.732$ & $4.866 / 70.382$ \\
\hline Independent reflections & 1468 & 4262 \\
\hline$R_{\text {int }}$ & 0.0552 & 0.0649 \\
\hline$F(000)$ & 314.0 & 628.0 \\
\hline Restraints/parameters & $0 / 37$ & $0 / 105$ \\
\hline Goodness-of-fit on $F^{2}$ & 1.023 & 1.051 \\
\hline$R_{1} / \mathrm{w} R_{2}[I \geq 2 \sigma(I)]$ & $0.0395 / 0.0795$ & $0.0551 / 0.1340$ \\
\hline 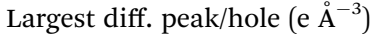 & $1.39 /-0.97$ & $1.46 /-1.30$ \\
\hline
\end{tabular}


Table 2 Bond lengths and angles for the $\left[\mathrm{CuBr}_{2}\right]_{n}$ magnetic chains of 1 at $300 \mathrm{~K}$ and $240 \mathrm{~K}$

\begin{tabular}{lll}
\hline & $300 \mathrm{~K}$ & $240 \mathrm{~K}$ \\
\hline Bond lengths & & \\
$\mathrm{Cu} 1-\mathrm{Br} 1$ & $2.3813(5) \AA$ & $2.37(1) \AA$ \\
$\mathrm{Br} 1-\mathrm{Br} 2$ & $3.593(2) \AA$ & $3.80(1) \AA$ \\
$\mathrm{Cu} 2-\mathrm{Br} 2$ & $2.3813(5) \AA$ & $2.38(6) \AA$ \\
$\mathrm{Cu} 2-\mathrm{Br} 3$ & $2.3813(5) \AA$ & $2.37(1) \AA$ \\
$\mathrm{Br} 3-\mathrm{Br} 4$ & $3.593(2) \AA$ & $3.80(1) \AA$ \\
$\mathrm{Cu} 3-\mathrm{Br} 4$ & $2.3813(5) \AA$ & $2.38(6) \AA$ \\
& & \\
Bond angles & & $156.51(2)^{\circ}$ \\
$\mathrm{Cu} 1-\mathrm{Br} 1-\mathrm{Br} 2$ & $180.0^{\circ}$ & $167.20(6)^{\circ}$ \\
$\mathrm{Br} 1-\mathrm{Br} 2-\mathrm{Cu} 2$ & $179.84(9)^{\circ}$ & $175.09(9)^{\circ}$ \\
$\mathrm{Br} 2-\mathrm{Cu} 2-\mathrm{Br} 3$ & $179.69(4)^{\circ}$ & $156.51(1)^{\circ}$ \\
$\mathrm{Cu} 2-\mathrm{Br} 3-\mathrm{Br} 4$ & $180.0^{\circ}$ & $167.20(6)^{\circ}$ \\
$\mathrm{Br} 3-\mathrm{Br} 4-\mathrm{Cu} 3$ & $179.84(9)^{\circ}$ &
\end{tabular}

Such a discontinuous jump at $T_{\mathrm{S}}$ can not be attributed to a second-order transition but arises from a first-order structural transition, as also evidenced by our X-ray diffraction results which will be shown later. At lower temperatures a maximum appears at around $50 \mathrm{~K}$ and then decreases, a typical behavior for one-dimensional magnets. The maximum temperature is consistent with the results of Inman et al. which is $47 \mathrm{~K}^{2}$ We used the Bonner-Fisher formula for a Heisenberg magnetic chain to approximately describe the magnetic susceptibility of the LT phase. ${ }^{18}$ The fitting is represented by the red solid line in Fig. 1(a) which gives $J_{\mathrm{L}} / k_{\mathrm{B}}=-88.18 \pm 0.89 \mathrm{~K}$ and $g=2.40 \pm$ 0.01 .

The magnetization at $2 \mathrm{~K}$ as a function of applied field is shown in Fig. 1(c) in which $M$ varies quasi-linearly with $H$ up to 7 $\mathrm{T}$. Especially, the measured magnetization at $7 \mathrm{~T}$ is only $0.25 \mu_{\mathrm{B}} /$ $\mathrm{Cu}^{2+}$, far smaller than the spin-only saturated value $1.73 \mu_{\mathrm{B}} / \mathrm{Cu}^{2+}$ of $\mathrm{Cu}^{2+}$ with spin $-1 / 2$. This behavior again indicates strong antiferromagnetic coupling in $\mathbf{1}$.

The crystal structures of 1 were solved at $300 \mathrm{~K}$ and $240 \mathrm{~K}$. The refinement results are listed in Table 1 . The chemical formula of 1 is $\left(\mathrm{C}_{6} \mathrm{H}_{8} \mathrm{~N}_{2}\right) \mathrm{CuBr}_{2}$ which can also be verify by the thermogravimetric analysis measurement (see ESI $\dagger$ ) and elemental analysis. As can be seen in Fig. 2(a), at $300 \mathrm{~K}$ the space group of 1 is Pmmn with the lattice parameters $a=8.3552(9) \AA$, $b=8.5782(11) \AA$, and $c=6.6959(7) \AA$. The structure of 1 is similar to its analogous coordination compound $\mathrm{Cu}(2,5$-dimethylpyrazine) $\mathrm{Br}_{2}$ (ref. 15) with - $\mathrm{Cu}-2,6$-dimethylpyrazine- $\mathrm{Cu}-$ infinite structure along the $c$ axis. The copper(II) atom is tetrahedrally coordinated by two bromide atoms and two $\mathrm{N}$ atom from the 2,5-dimethylpyrazine ring. The $\mathrm{Cu}-\mathrm{Br}$ bond length is $2.381 \AA$, and the Cu-N bond lengths are 1.976-1.982 $\AA$ (right panel of Fig. 2(a)). The linear $\mathrm{Cu}-\mathrm{Br} \cdots \mathrm{Cu}$ magnetic chains propagate along the $a$ axis with the non-bonding two-halide contacts ( $\mathrm{Br} \cdots \mathrm{Br}$ length $d_{\mathrm{Br} \cdots \mathrm{Br}}=3.593 \AA$, $\mathrm{Cu}-\mathrm{Br} \cdots \mathrm{Br}$ angle $=$ $\left.180.0^{\circ}\right)$. The mean plane of 2,6-dimethylpyrazine ring perpendicular to the chain direction. In view that $d_{\mathrm{Br} \cdots \mathrm{Br}}=3.632 \AA$ in $\mathrm{Cu}\left(2,5\right.$-dimethylpyrazine) $\mathrm{Br}_{2},{ }^{15}$ both compounds should also have similar intra-chain super-exchange strength.
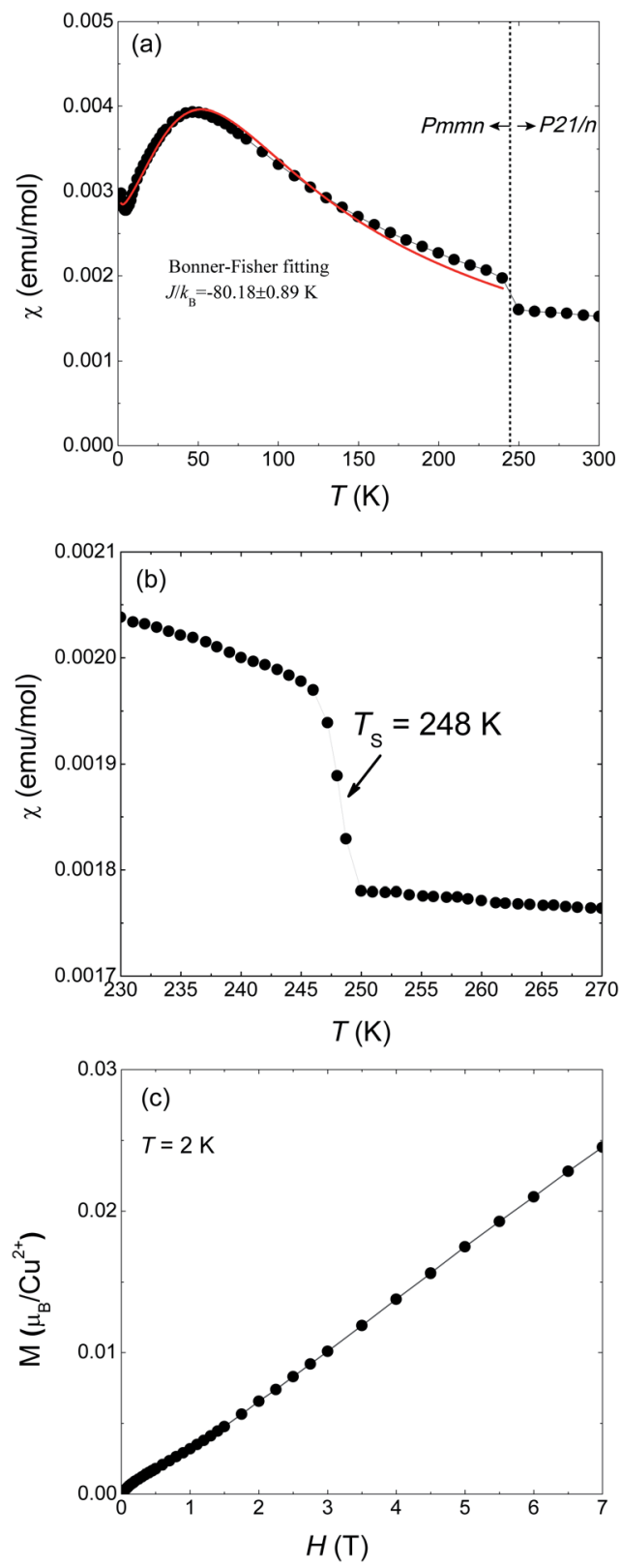

Fig. 1 Magnetic susceptibility and magnetization of $\mathrm{Cu}(2,6$-dimethylpyrazine) $\mathrm{Br}_{2}$ powdered sample. (a) Magnetic susceptibility in temperature range $2-300 \mathrm{~K}$ under an applied field of $H=1000 \mathrm{Oe}$. (b) The jump of $\chi$ at $T_{\mathrm{S}}=248 \mathrm{~K}$ corresponds to the orthorhombic-tomonoclinic structure phase transition. (c) Magnetization data as a function of field up to $7 \mathrm{~T}$ at $T=2 \mathrm{~K}$.

However, the structure of $\mathbf{1}$ at $240 \mathrm{~K}$ dramatically changes to the monoclinic type with $P 21 / n$ structure. At the LT phase, the $\mathrm{Cu}-\mathrm{N}$ and $\mathrm{Cu}-\mathrm{Br} 2$ bond lengths were enlarged to 1.989-1.991 and $2.386 \AA$, respectively. Only the $\mathrm{Cu}-\mathrm{Br} 1$ bond length was shortened to $2.371 \AA$. The 2,6-dimethylpyrazine rings also display rotation around the $\mathrm{N} 1-\mathrm{Cu}-\mathrm{N} 2$ axis (right panel of Fig. 2(b)). The two-halide contacts are obviously distorted to form a non-linear spin chain $\left(\mathrm{Br} \cdots \mathrm{Br}\right.$ length $d_{\mathrm{Br} \cdots \mathrm{Br}}=3.80(1) \AA$, $\mathrm{Cu}-\mathrm{Br} \cdots \mathrm{Br}$ angle $\left.=156.51(2)^{\circ}\right)$. These large lattice distortions across $T_{\mathrm{S}}$ make the $\left[\mathrm{CuBr}_{2}\right]_{n}$ magnetic chains substantially 
(a)

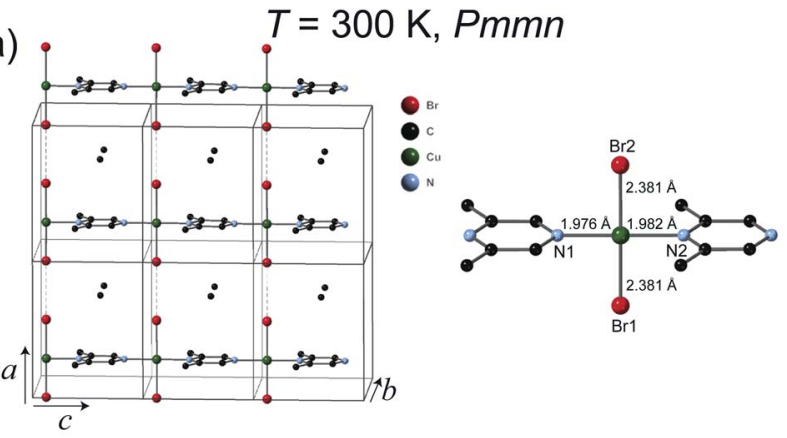

(b)

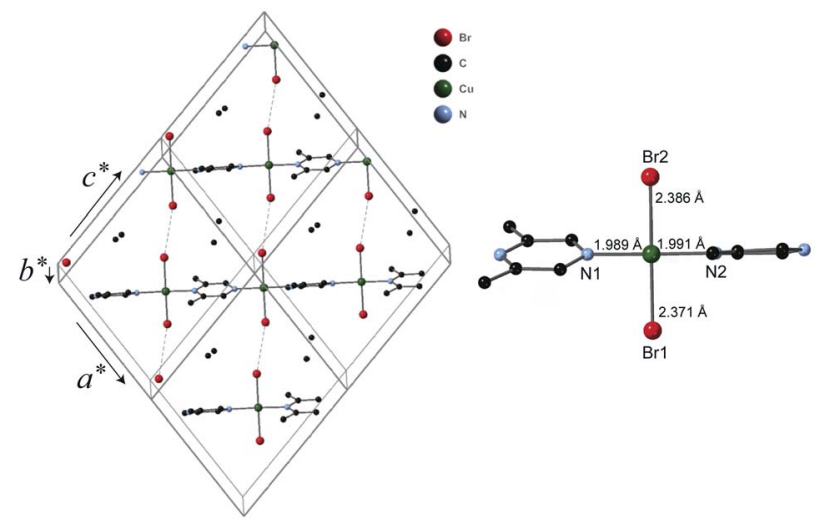

Fig. 2 Crystal structures of 1. (a) The high temperature (HT) orthorhombic structure phase $(T=300 \mathrm{~K})$. (b) The low temperature (LT) monoclinic structure phase $(T=240 \mathrm{~K})$. The coordination environment of copper(II) is shown in the right panel. Hydrogen atoms are omitted for clarity.

twisted, rendering the crystal to a lower symmetry. The $\left[\mathrm{CuBr}_{2}\right]_{n}$ chain structure and its detailed length/angle parameters at 300 and $240 \mathrm{~K}$ are shown in Fig. 3 and Table 2.

The observation of structural phase transition in $\mathbf{1}$ is surprising because it has never been found in its analog compounds like $\mathrm{Cu}(2,5$-dimethylpyrazine $) \mathrm{Br}_{2}, \quad \mathrm{Cu}(2,6-$ dimethylpyrazine) $\left(\mathrm{NO}_{3}\right)_{2}$, or other pyrazine-based coordination

(a) $T=300 \mathrm{~K}$

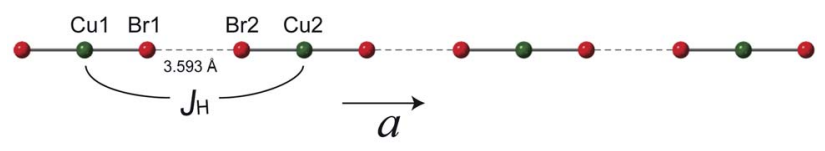

(b) $T=240 \mathrm{~K}$

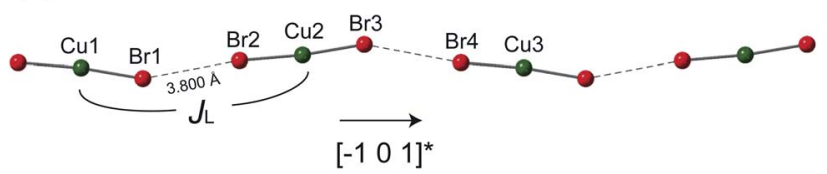

Fig. 3 The $\left[\mathrm{CuBr}_{2}\right]_{n}$ magnetic chains at (a) $300 \mathrm{~K}$ and (b) $240 \mathrm{~K}$. The antiferromagnetic super-exchanges between neighboring copper ions through the $\mathrm{Br} \cdots \mathrm{Br}$ non-bonding contact are denoted as $\mathrm{J}_{\mathrm{H}}$ and $J_{L}$ for the HT and LT phases, respectively. The detailed bond lengths and bond angles of the spin chains are listed in Table 2. complexes at ambient pressure., ${ }^{2,15,19-21}$ The linear to non-linear structural transition of $\left[\mathrm{CuBr}_{2}\right]_{n}$ chains bring substantial change in the coordination environment and should have considerable impact on its 1D magnetism.

It is known that superexchange through the pyrazine ring pathway arises from the weak coupling $\pi$ mechanism and the strength is less than $J / k_{\mathrm{B}}=-10.3 \mathrm{~K}$ is all cases. ${ }^{14}$ For example, the 2,6-dimethylpyrazine bridged exchange strength in $\mathrm{Cu}(2,6-$ dimethylpyrazine $\left(\mathrm{NO}_{3}\right)_{2}$ is only $J=-4.0 \pm 0.1 \mathrm{~K},{ }^{14}$ far smaller than the exchange strength in $\mathbf{1}$. So the dominate exchange in $\mathbf{1}$ through the-Cu-2,6-dimethylpyrazine-Cu-pathway is unlikely. Butcher et al. investigated the structure and magnetic behavior of $\mathrm{Cu}$ (2,5-dimethylpyrazine) $\mathrm{Br}_{2}$ combined with quantum Monte Carlo simulations. ${ }^{15}$ They found the strong antiferromagnetic exchange $J=-234 \mathrm{~K}$ is through the $\mathrm{Cu}-\mathrm{Br} \cdots \mathrm{Br}-\mathrm{Cu}$ pathway, whereas the $\mathrm{Cu}-2,5$-dimethylpyrazine-Cu pathway only generates a much weaker strength $J^{\prime}=-12 \mathrm{~K}$. In sight its similar structure to 1 , it is appropriate to attribute the propagation of $J_{\mathrm{L}}$ to the $\mathrm{Cu}-\mathrm{Br} \cdots \mathrm{Br}-\mathrm{Cu}$ pathway.

The buckling of $\left[\mathrm{CuBr}_{2}\right]_{n}$ spin chain in $\mathbf{1}$ across $T_{\mathrm{S}}$ offers an unprecedented opportunity to investigate the relation of chain geometry with 1D magnetism. In the HT phase, the closest $\mathrm{Br} \cdots$ $\mathrm{Br}$ is $3.593 \AA$ which is very short in comparison with the chain structure in strong coupled tetrabromocuprate compounds., ${ }^{1,22}$ Together with the linear chain structure, the $\mathrm{Cu}-\mathrm{Br} \cdots \mathrm{Br}-\mathrm{Cu}$ pathway is expected to propagate strong antiferromagnetic exchange. At the $\mathrm{LT}$ phase, the $\mathrm{Cu}-\mathrm{Br} \cdots \mathrm{Br}$ angle and the $\mathrm{Cu}-$ $\mathrm{Br} \cdots \mathrm{Br}-\mathrm{Cu}$ dihedral angle all significantly deviate from $180^{\circ}$. According to the Goodenough-Kanamori rules, non-linear pathway propagate weaker superexchange. ${ }^{23}$ Also, the $\mathrm{Br} \cdots \mathrm{Br}$ contact distance is enlarged to $3.800 \AA$, this would further reduce the intra-chain exchange strength. ${ }^{1}$ The analysis is evidenced by the jump at $T_{\mathrm{S}}$ in Fig. 1(a). However, a qualitative theory should consider all geometric variables in the chain structure which is not currently available.

Fig. 4 is the specific heat data from 100 to $2 \mathrm{~K}$ at zero field. $C$ monotonously decreases with lowering temperature. The

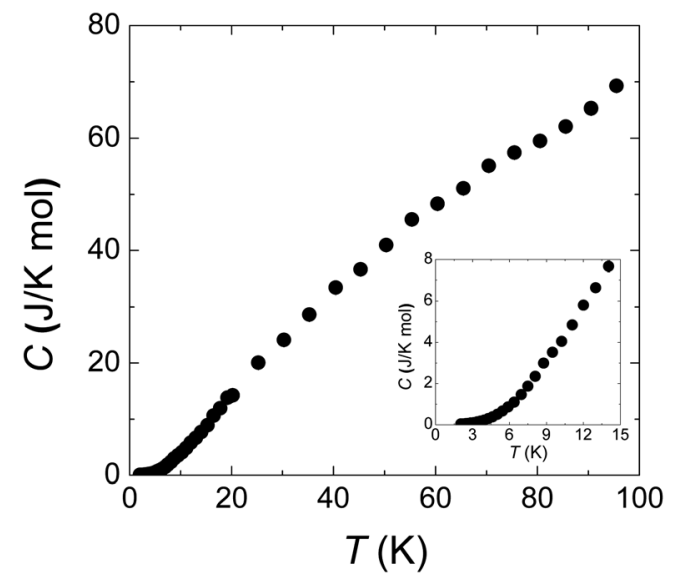

Fig. 4 Specific heat from 2 to $100 \mathrm{~K}$. The inset panel shows the data below $15 \mathrm{~K}$. The smooth curve indicates the absence of long range spin ordering down to $2 \mathrm{~K}$. 
absence of any anomaly in the measured temperature range verifies the broad peak around $50 \mathrm{~K}$ in $\chi$ (Fig. 1(a)) is purely from one-dimensional magnetism, not magnetic ordering. There is also no sign of magnetic ordering transition down to $2 \mathrm{~K}$ in specific heat data (inset of Fig. 4).

The optical properties were investigated by PL and UV-vis absorption spectra measurements (Fig. 5). The PL spectrum was excited by a $325 \mathrm{~nm}$ laser source and the maximum wavelength is at $445 \mathrm{~nm}$, as shown by the black dots in Fig. 6. This PL peak wavelength corresponds to an electron band gap of $2.79 \mathrm{eV}$. The PL emission peak of $445 \mathrm{~nm}$ can be assigned to the $\pi-\pi^{*}$ transition of between the organic ligand. The UV-vis absorption spectrum shows an obvious Stokes shift effect with a maximum appearing at around $280 \mathrm{~nm}$.

To get further insight on its electric and optical properties, we theoretically calculated the electronic structure and optical properties of 1 by using the Vienna $a b$ initio simulation package (VASP) 6.1.2 code with a projector-augmented-wave pseudopotential. $^{\mathbf{2 4 - 2 6}}$ The exchange-correlation functional for describing the electron interactions is the generalized gradient approximation with Perdew-Burke-Ernzerh of parametrization (GGAPBE). ${ }^{27}$ The Heyd-Scuseria-Ernzerhof (HSE06) hybrid functional $^{28}$ is adopted to calculate the electronic properties, optical properties, and mobility calculations because of the GGA-PBE functional usually underestimate the band energy gap.

There are 38 atoms in the unit cell with the Pnmn structure. The $3 \times 3 \times 3 \Gamma$-centered $k$-mesh of Brillouin zone (BZ) are employed in integration, but the band structure are calculated with additional 7 special $k$ points including 71 points. The energy cutoff the plane-wave basis sets is $500 \mathrm{eV}$ to ensure the calculations to reach convergence. The energy convergent criterion is $10^{-6} \mathrm{eV}$. In the relaxation calculation, all the force is smaller than $0.01 \mathrm{eV}^{-1}$ for the equilibrium structures.

The optical absorption coefficient $\alpha(\omega)$ is used to quantitatively describe the response-ability of 1. $\alpha(\omega)$ can be determined by the imaginary part $\varepsilon_{2}(\omega)$ of the complex dielectric function $\varepsilon(\omega)=\varepsilon_{1}(\omega)+\mathrm{i} \varepsilon_{2}(\omega)$. In the present work $\varepsilon_{2}(\omega)$ is calculated by the following equation ${ }^{29-31}$

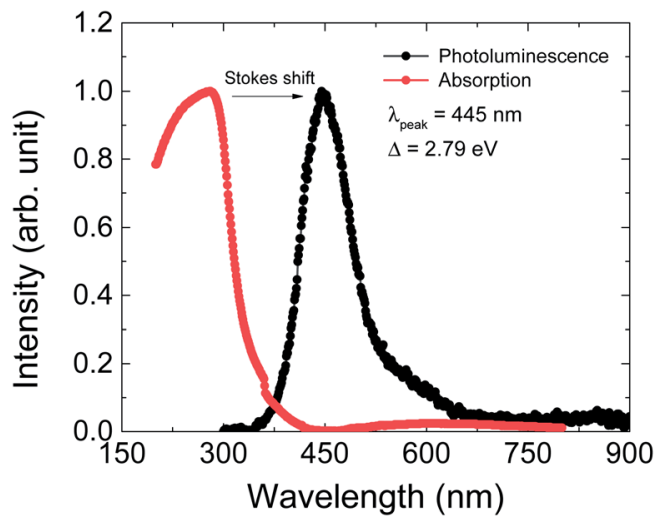

Fig. 5 Photoluminescence (PL, black dots) and UV absorption (red dots) spectra of $\mathrm{Cu}\left(2,6\right.$-dimethylpyrazine) $\mathrm{Br}_{2}$ at room temperature. The excitation wavelength for $\mathrm{PL}$ is $325 \mathrm{~nm}$ and a strong peak appears at $445 \mathrm{~nm}$, corresponding to an energy gap of $\Delta=2.79 \mathrm{eV}$.
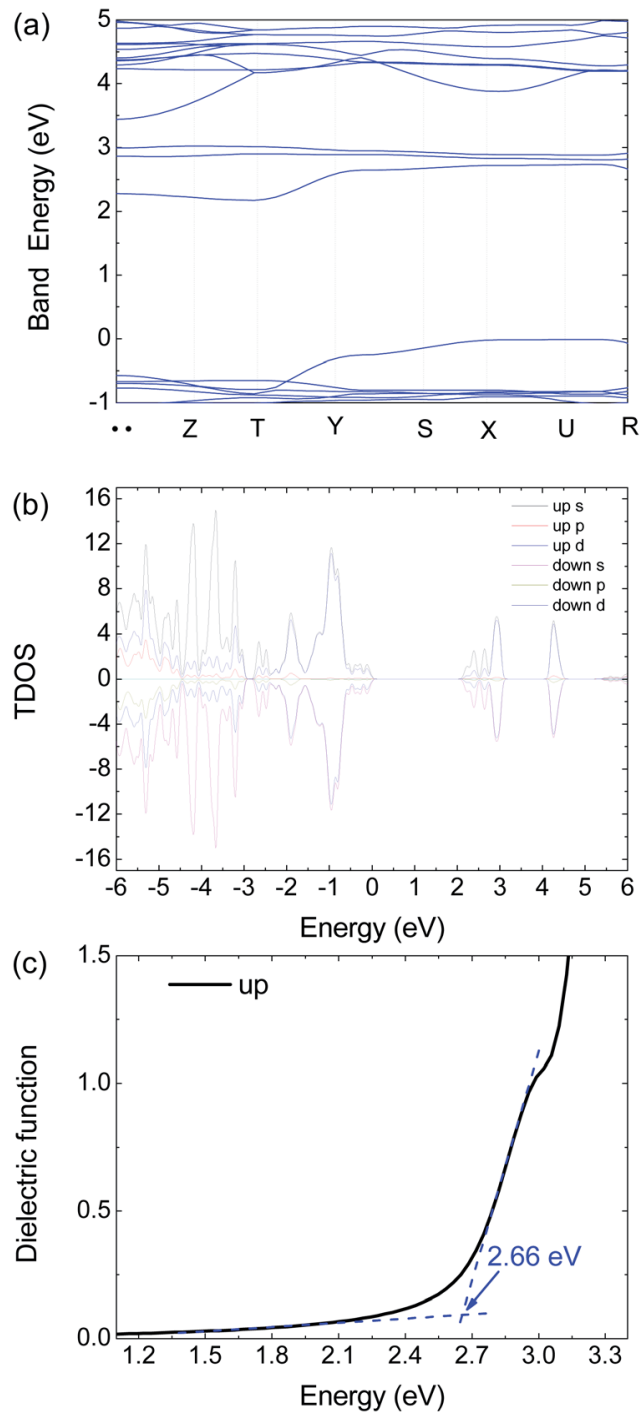

Fig. 6 Calculated (a) electronic band structure, (b) TDOS and (c) dielectric function of 1 by HSE06 with an antiferromagnetism model.

$$
\varepsilon_{2}(\omega)=\frac{4 \pi^{2}}{m^{2} \omega^{2}} \sum_{\mathrm{c}, \mathrm{v}} \int_{\mathrm{BZ}} \frac{2}{(2 \pi)^{3}}\left|M_{\mathrm{c}, \mathrm{v}}(k)\right|^{2} \delta\left(\varepsilon_{\mathrm{ck}}-\varepsilon_{\mathrm{vk}}-h \omega\right) d^{3} k
$$

where $\left|M_{\mathrm{c}, \mathrm{v}}(k)\right|^{2}$ is the momentum matrix elements. The conduction and valence band states are represented by $\mathrm{c}$ and $\mathrm{v}$. While $\alpha(\omega)$ via the following expression

$$
\alpha(\omega)=\sqrt{2} \omega \sqrt{\sqrt{\varepsilon_{1}^{2}(\omega)+\varepsilon_{2}^{2}(\omega)}-\varepsilon_{1}(\omega)}
$$

where the real part $\varepsilon_{1}(\omega)$ is calculated from the imaginary part $\varepsilon_{2}(\omega)$ of the complex dielectric function by using the KramerKronig relationship. $\varepsilon_{2}(\omega)$ is calculated by using the HSE06 functional. The calculated band structure and total density of states (TDOS) with obital characteristics of $\mathbf{1}$ are presented in Fig. 6(a and b). The calculated electronic band gap can be estimated from the dielectric function curve as presented in Fig. 6(c) which is $2.66 \mathrm{eV}$, in good agreement with the experimental value $2.79 \mathrm{eV}$ from our PL measurement. 


\section{Conclusions}

In summary, we synthesized single crystalline $\mathrm{Cu}(2,6$-dimethylpyrazine) $\mathrm{Br}_{2}$ and successively solved its structure. The linear $\left[\mathrm{CuBr}_{2}\right]_{n}$ chains demonstrate strong one-dimensional antiferromagnetism via the two-halide exchange pathway, similar to its analog compound $\mathrm{Cu}(2,5$-dimethylpyrazine $) \mathrm{Br}_{2}$. However, $\mathrm{Cu}$ (2,6-dimethylpyrazine) $\mathrm{Br}_{2}$ experiences a special phase transition from Pmmn to $P 21 / n$ structure at $T_{\mathrm{S}}=248 \mathrm{~K}$. The $\left[\mathrm{CuBr}_{2}\right]_{n}$ spin chains are non-linear and the average antiferromagnetic exchange strength get weakened after transition to the low temperature phase. Thus our experiments reveal a novel onedimensional antiferromagnet with linear to non-linear structural transition in the spin chains and provide an example compound in the study of two-halide exchange mechanism.

\section{Conflicts of interest}

There are no conflicts to declare.

\section{Acknowledgements}

This work is supported by the National Natural Science Foundation of China (Grant No. 11804137, 62075092, 11874192) and the Natural Science Foundation of Shandong Province (Grant No. ZR2020YQ03, ZR2018BA026,ZR2019MA066).

\section{Notes and references}

1 M. M. Turnbull, C. P. Landee and B. M. Wells, Coord. Chem. Rev., 2005, 249, 2567-2576.

2 G. W. Inman and W. E. Hatfield, Inorg. Chem., 1972, 11, 3085-3090.

3 C. P. Landee and M. M. Turnbull, Eur. J. Inorg. Chem., 2013, 2266-2285.

4 H.-J. Mikeska and A. K. Kolezhuk, Lect. Notes Phys., 2004, 645, 1-83.

5 K. K. Sharma, R. Singh, N. Fahmi and R. Singh, J. Coord. Chem., 2010, 63, 3071-3082.

6 R. Block and L. Jansen, Phys. Rev. B: Condens. Matter Mater. Phys., 1982, 26, 148-153.

7 P. Straatman, R. Block and L. Jansen, Phys. Rev. B: Condens. Matter Mater. Phys., 1984, 29, 1415-1418.

8 L. O. Snively, G. F. Tuthill and J. E. Drumheller, Phys. Rev. B: Condens. Matter Mater. Phys., 1981, 24, 5349-5355.

9 M. B. Stone, D. H. Reich, C. Broholm, K. Lefmann, C. Rischel, C. P. Landee and M. M. Turnbull, Phys. Rev. Lett., 2003, 91, 037205.

10 T. Lancaster, S. J. Blundell, M. L. Brooks, P. J. Baker, F. L. Pratt, J. L. Manson, C. P. Landee and C. Baines, Phys. Rev. B: Condens. Matter Mater. Phys., 2006, 73, 020410.
11 H. Kühne, A. A. Zvyagin, M. Günther, A. P. Reyes, P. L. Kuhns, M. M. Turnbull, C. P. Landee and H.-H. Klauss, Phys. Rev. B: Condens. Matter Mater. Phys., 2011, 83, 100407.

12 H. Kühne, H.-H. Klauss, S. Grossjohann, W. Brenig, F. J. Litterst, A. P. Reyes, P. L. Kuhns, M. M. Turnbull and C. P. Landee, Phys. Rev. B: Condens. Matter Mater. Phys., 2009, 80, 045110.

13 A. V. Sologubenko, K. Berggold, T. Lorenz, A. Rosch, E. Shimshoni, M. D. Phillips and M. M. Turnbull, Phys. Rev. Lett., 2007, 98, 107201.

14 H. W. Richardson and W. E. Hatfield, J. Am. Chem. Soc., 1976, 98, 835-839.

15 R. T. Butcher, J. J. Novoa, J. Ribas-Ariño, A. W. Sandvik, M. M. Turnbull, C. P. Landee, B. M. Wells and F. F. Awwadi, Chem. Commun., 2009, 1359-1361.

16 O. V. Dolomanov, L. J. Bourhis, R. J. Gildea, J. A. K. Howard and H. Puschmann, J. Appl. Crystallogr., 2010, 42, 339-341.

17 G. M. Sheldrick, Acta Crystallogr., Sect. C: Struct. Chem., 2015, 71, 3-8.

18 J. C. Bonner and M. E. Fisher, Phys. Rev., 1964, 135, A640A658.

19 B. R. Jones, P. A. Varughese, I. Olejniczak, J. M. Pigos, J. L. Musfeldt, C. P. Landee, M. M. Turnbull and G. L. Carr, Chem. Mater., 2001, 13, 2127-2134.

20 F. F. Awwadi, C. P. Landee, M. M. Turnbull, B. Twamley and B. M. Wells, Polyhedron, 2005, 24, 2153-2159.

21 J. C. Monroe, C. P. Landee, M. M. Turnbull, M. Polson and J. L. Wikaira, Polyhedron, 2019, 171, 344-352.

22 J. L. Wikaira, L. Li, R. Butcher, C. M. Fitchett, G. B. Jameson, C. P. Landee, S. G. Telfer and M. M. Turnbull, J. Coord. Chem., 2010, 63, 2949-2964.

23 J. B. Goodenough, Magnetism and the Chemical Bond, Wiley, New York, 1963.

24 G. Kresse and J. Furthmüller, Phys. Rev. B: Condens. Matter Mater. Phys., 1996, 54, 11169-11186.

25 P. E. Blöchl, Phys. Rev. B: Condens. Matter Mater. Phys., 1994, 50, 17953-17979.

26 G. Kresse and D. Joubert, Phys. Rev. B: Condens. Matter Mater. Phys., 1999, 59, 1758-1775.

27 J. P. Perdew, K. Burke and M. Ernzerhof, Phys. Rev. Lett., 1996, 77, 3865-3868.

28 J. Heyd, G. E. Scuseria and M. Ernzerhof, J. Chem. Phys., 2003, 118, 8207-8215.

29 S. Saha, T. P. Sinha and A. Mookerjee, Phys. Rev. B: Condens. Matter Mater. Phys., 2000, 62, 8828-8834.

30 X. Zhang, X. Zhao, D. Wu, Y. Jing and Z. Zhou, Adv. Sci., 2016, 3, 1600062 .

31 P. Li, C. wen Zhang, J. Lian, M. juan Ren, P. ji Wang, X. hong Yu and S. Gao, Opt. Commun., 2013, 295, 45-52. 